\section{A Climiral lerture}

\section{ON

FRACTURES OF THE BASE OF
THE SKULL.

Delivered at St. Bartholomew's Hospital.

BY ANTHONY BOWLBY, C.M.G., F.R.C.S., SURGEON TO THE HOSPITAL.

Gentlemen,-Some of you remember the case of a woman who was in Stanley Ward two or three weeks ago, and whom we believed to have fracture of the base of the skull. She had met with an injury, a blow which knocked her backwards so that she fell against the kerbstone, and was admitted more or less collapsed, though not unconscious. She had been bleeding from the ear, and subsequently she had slight facial paralysis. She never became unconscious, nor at any time was she maniacal or lost control of her actions. The facial paresisfor it was not an actual paralysis-gradually improved, until practically it had all disappeared. She had no deafness, or any discharge of cerebrospinal fluid, or of any other fluid, from her ear. She gradually got quite well. That was an instance of fracture of the base of the skull, uncomplicated except by slight facial paresis. A little before that date there was admitted into the hospital a man who had met with an injury. When he was admitted he was also conscious. He had a discharge of blood from his ear, and examination of the ear showed that his membrana tympani was torn.' 'His condition became worse; he got gradually stupid and heavy, and then, as I understand, because I did not see him at this time, he became slowly more and more unconscious, took his food badly, was more and more difficult to arouse from his state of stupor, and ultimately he passed into a state of coma, in which he died. Examination showed that he had fracture of the left side of the skull at its base, across the temporal bone. There was some haemorrhage between the dura mater and the bone, and there was a tear of his temporo-sphenoidal lobe, which happened to be on the side opposite to the injury. There was also a haemorrhage underneath the pia mater, so that there was an extravasation of blood over the surface of his brain. That, you see, was a case of fracture of the base of the skull complicated by definite severe injury to the brain.

Now in all fractures of the base it may be said in general terms that the diagnosis of a breaking of a particular portion of bone depends on three things-the escape of blood, the escape of cerebro-spinal fluid, and injuries to nerves; and where there is any part of the base of the skull broken it is to those three conditions you look for evidence as to the injury. When the middle fossa is broken it is ordinarily fractured across the petrous portion of the temporal bone, and whether there are complications or not depends very much on the line of this fracture: Supposing that the line of fracture does not involve any part of the bony canal to the seventh pair of nerves, then under those circumstances there is ordinarily no escape of cerebro-spinal fluid, no evidence of nerve injury. So the bone may be broken and the only evidence of that break may be an escape of blood through the external auditory meatus. But there may not be even that evidence, because unless the membrana tympani is torn the blood cannot escape. So you see it is quite possible for a person to have fracture of the middle fossa of the base of the skull and for all these three symptoms -the escape of blood, the escape of cerebro-spinal fluid, and injuries of 'nerves-to be absent; and it is very diffi? cult to say positively that a person has not a fracture of the base of the skull. We will go a step further and ask under what circumstances does the cerebro-spinal fluid escape? It can only escape when the dura mater or arachnoid are torn. Where are they torn? They are practically always torn where they are reflected around the seventh pair of nerves as they pass into the internal auditory meatas. And if the dura mater and arachnoid are torn and the bone is broken and the membrans tympani is torn, there is of contse a cammunication opéned up between the subarachnold spoce on one side and the external auditory meatus on the other. And in proportion as that is a wide tear, the fluid can easily filter out through the damaged portion of the skull.

The next thing to consider is, what sort of escape of fluid is it that is suggestive or characteristic of fracture of the base of the skull? All discharges of fluid from the external ear are not suggestive or indicative of fracture of the middle fossa and the escape of cerebro-spinal fluid. You must remember that the ear itself contains a mucous cavity, and that mucous cavity may secrete a considerable amount of fluid after injury. Fluid can be also poured out from the tympanic cavity or the semicircular canals, and the fluid may pass out through the fracture into the meatus. The result is that the mere escape of a small quantity of fluid, perhaps mucus and of a sticky consistency, does not indicate fracture of the middle fossa. The characteristic discharge which points to escape of cerebro-spinal fluid is this: the discharge comes on very soon after the accident, and it either accompanies the flow of blood which is caused by the accident, or it immediately follows the cessation of that flow of blood. And the flow is copious. The discharge of mucous fluid from the tympanum or from some place inside the ear is never copious. What do I mean by copious? Because that, after all, is a relative term. A man was admitted into casualty ward some years ago with a fracture of the base of his skull. I saw him the next morning; and his pillow was wet, his bandages were wet, and the mattress was wet. If you had put a test tube underneath his ear you could have half-filled it in four or five minutes. He probably had soaked his bandages and his pillow and mattress with not less than a pint or two of fluid. That is what I should call a characteristically copious discharge of fluid, which could come from nowhere else but the subarachnoid space. In character the appearance of the fluid is not mucous, but quite clear; it is of low specific gravity, and, if you had enough of it to test, you could probably find traces of sugar, and possibly small quantities of chloride of sodium. It would have a specific gravity of about 1001 . That is the kind of escape which you meet with after an injury to the dura mater and arachnoid. Then, as regards injury to nerves. The nerves which are injured, or which may be injured in fractures of the middle fossa, are the facial and the auditory. In the woman of whom I have spoken the nerve injured was the one which usually escapes-namely, the facial. It is not often that the facial nerve is affected in fractures of the base of the skull. The auditory nerve, however, is frequently involved under these circumstances. This is softer and more easily torn, and another thing which is noticeable about it is that, when it is injured, it is much more difficult to get it to mend. On the other hand, in the majority of cases when the facial nerve is hurt, as it was in this woman, the facial paresis rapidly passes off; whereas the deafness which follows injury of the auditory nerve and the middle ear and the tinnitus which also follows it are liable to continue even for years after the accident. A man came into this hospital with a fracture of the middle fossa of his skull, and with deafness and tinnitus. He recovered from his injury, but he never recovered his hearing, and he always had, for the rest of his life, as far as I know, certainly during the five years that I followed him up, constant tinnitus, with a humming or whistling noise in the affected ear. This did not seem to get any better, though, as time went on, he took less notice of it than at first.

If all these symptoms which I have mentioned have been observed-the escape of blood, followed by an escape of clear fluid in large quantities and associated with evidence of injury to the auditory or facial nerve-you can say exactly what has happened. There is a fracture:across the petrous bone, which on the one side involves the tympanum, the membrana tympani being torn, and on the other side it extends inwards to some part of the canal of the seventh pair of nerves, and is associated with a tear in the dura mater and arachnoid. Thus you can diagnose exactly what portion of bone has been injured.

Now with regard to the anterior fossa. That may be broken in various ways. It may be by direct violence from the front, by hlows upon the frontal or naso-frontal region, and it may be broken by something being thrust into the orbit; stach as the end of an umbrella or $a$ wialk,ing-stick. and passing into the brain. But ordinari]y the fossa is broken by falls apon the forehead or upon the top 
of the head. A man falls from a height or is pitched out of a cab or off an omnibus, and lands on his frontal region and fractures the anterior fossa. There are, again, the same symptoms to look for: the escape of blood and of cerebro-spinal fluid and the injury to nerves.

First, with regard to the escape of blood. Blood ordinarily escapes in these cases in large quantities from the nose and from the back of the nose into the pharyns, and possibly into the stomach, and this blood which passes into the stomach may afterwards be vomited. And it is much more difficult to say in a case of a blow on the nasofrontal region that the blood comes from the injury to the base of the skull than it is when there is bleeding into the ear, because in the case of injury following a blow on the nose it is difficult to say whether the bleeding is from the nose or from the pharynx. It is only when the haemorrhage is very copious and continuous that you can say that it is the sort of haemorrhage which is likely to result not merely from a broken nose, but from fracture of some part of the ethmoid or sphenoid or both. Blood may collect in the orbit, and it is very difficult to say for certain that escape of blood into the orbit is certainly due to fracture of the bone of the anterior part of the fossa. But when the blood accumulates underneath the conjunctiva posteriorly, that is very suggestive of percolation of blood from the back of the orbit, and is unlike the ordinary extravasation causing a black eye. As regafds an escape of cerebro-spinal fluid, I have only seen one case in which there has been a considerable quantity of clear fluid discharged into the nose. You will readily see the difficulty. When a man is lying down any fluid tends to pass down through the back of the nose into the pharynx, and nobody knows of it. Cerebro-spinal fluid may be discharged in these cases without anybody knowing that it is being discharged, and, as it mixes with the secretions of the nose, it mostly passes into the pharynx. So it has not the same importance as a discharge of fluid from the ear.

Lastly, there are the injuries to nerves. Any one of the first six nerves may be injured. In a case of fracture of the anterior fossa at the base of the skull a man may have a tear of his optic nerve and blindness, but if he has an injury to his olfactory nerve no one knows of it at the time of the accident because it cannot be tested. He may also have a squint, due to injury to the motor nerves of the eye, so there may be evidence of implication of nerves suggesting fracture of the anterior fossa of the skull. Therefore, in a case of such fracture the diagnosis to some extent depends upon the evidence of injury of nerves. But here, as in the case of the facial, the injury to nerves is usually not so serious as to prevent quick recovery. So that these patients, as a rule, have rapid restoration of function where the nerves have been more or less seriously injured.

I want to mention one other fossa before we pass on to speak of various complications, namely, the posterior fossa. The posterior fossa of the base of the skull is much less frequently injured than the others, and when it is injured it is usually broken by very great violence. This part of the bone is exceedingly thick and dense, and generally speaking a fracture of the posterior fossa is discovered rather at the post-mortem examination than by any clinical examination at the bedside, and the diagnosis of it is more difficult, because there is no external place from which the blood can discharge, and there is no external aperture through which the cerebro-spinal fluid can escape. Consequently, if a man has a fracture of the posterior fossa the blood accumulates in the tissues of the neck, and fracture may be suspected if there is a large collection of blood amongst the tissues attached to the occipital bone. But that might occur without fracture, so that the evidence which it affords of fracture having occurred is small. Injuries of nerves in fracture of the posterior fossa are rare, but in one case a patient was admitted here who had an injury to his head due to a fall out of a window. He had a large extravasation of blood into the tissues at the back of the neck and experienced great difficulty in swallowing food, though he could masticate it, because the pharyngeal muscles refused to act. He had considerable difficulty of respiration, and had attacks of interference with the heart's action. Three days later he died. We found at the autopsy that he had a fracture extending across his jugular foramen and involving the nerves there, and to an injury of the glosso- pharyngeal and the pneumogastric the symptoms were evidently due. But that is not at all a usual complication. So that in fractures of the posterior fossa all characteristic symptoms may be absent. There may be no definite escape of blood or cerebro-spinal fluid and no evident injury of nerves to tell you what portion of the bone of the skull is broken.

Now, think for a moment how it is and why it is that a certain number of these patients die, and a certain number, like the woman I have mentioned, recover. They die in proportion to the occurrence of complications. The breaking of a piece of bone at the base of the skull is not a fatal thing in itself. Why should a person die because he has a fracture across his petrous bone? Or what is there in a fracture of the occipital bone which should cause the person's death? Or why should a fracture of the ethmoid kill? These fractures in themselves are not the cause of death, and if there were no complications, the patients with these injuries would recover. Now the most important early complication is haemorrhage. Haemorrhage may be so severe that the mere loss of blood externally may be enough to kill the patient. You must remember that these fractures may be associated with tears of the large venous sinuses at the base of the skull. It is when these are torn, and occasionally when a large artery such as the carotid is torn, that the quantity of blood that is lost proves rapidly fatal. And that is not a case of a mere fracture itself killing the patient; the patient is killed because the fractured bone has torn a large blood vessel. And when a large quantity of blood is lost in this way death may take place exceedingly rapidly indeed, a patient may die in a few minutes who has had fracture of the base of the skull.

The next complication is the escape of blood inside the skull. That was present in the case of the man who died fifteen days after the accident, and who had extravasation of blood between his dura mater and the bone. So a patient may die from loss of blood externally, or from blood being poured out of his vessels into the cavity of the cranium, and so compressing the brain, or setting up irritation of the brain.

Still speaking of the complications of the fracture itself, the next thing you have to realize is that these fractures are often compound - that is, fractures in which the bone is exposed to the external air, and in which there is a tear opening up a region which is sometimes very difficult to keep aseptic. For instance, the external meatus is not always very clean, and, if there is a fracture and a tear of the membrana tympani, that fracture is in direct communication with the external ear. Under those circumstances there is a danger of sepsis, and this may spread through to the bone, and from the bone to the torn meninges. And such a patient may have septic meningitis, or he may develop an abscess of the brain, the brain having been, perhaps, also injured at the time of the accident. So here is a compound fracture, and it has to be treated as such. The external auditory meatus has to be rendered as aseptic as possible by washing it and cleaning it out, and it has to be dressed with some antiseptic preparation and if blood and cerebro-spinal fluid are escaping and soaking the dressings, the dressings will have to be changed, and every care must be devoted to preventing sepsis as far as possible. But think how little you can do when the fracture extends into the upper part of the pharynx or nose. You can do practically nothing; you cannot apply dressings to this part of the body, and the result is that in cases of fracture of the anterior fossa you have really to trust to chance whether there is or is not an infection of the broken bone from the cavity of the nose or the pharynx. Fortunately in a large number of cases there is no.infection, but septic infection may spread to the meninges and set up meningitis. In fracture of the posterior fossa this complication does not ordinarily occur, because there is not a communication with the outside and the fracture is not compound.

Apart from these complications, which may cause the man's death very quickly from bleeding or from sepsis, the chief complication of a fractured base is an injury to the brain, and the danger that awaits a large number of patients with fractures of the base of the skull is due not so much to the injury of bone as to the associated injury of the contents. of the skull. When a man has so severe an injury to his skull as to break it he is very likely to have as a result of that same injury a laceration of the 
brain. And a large number of these patients die, now from the bone trouble, not from the complications of the fracture itself, but from the associated injuries to the brain, which are not necessarily due to the fracture, but to the same cause which produced the fracture. As a rule it is not the broken bone which hurts the brain, it is the injury that broke the bone which also hurts the brain. In the case of a man who died in September, the injury of the bone was on one side, and the tear in the brain was on the other. The injury may be in any part, but is very likely to be somewhere at the base, for the parts of the brain which are most likely to be torn are the parts which lie in contact with the rough bone of the anterior and middle fossae of the skull. Look inside a skull in the post-mortem room and see what it is that the brain rests upon, apart from the meningeal fluid which is present. The frontal lobes lie upon the rough,irregular, lumpy, orbital plates of the frontal bone. And there is immediately behind that the sphenoid and ethmoid, with their projecting osseous prominences, and when the brain is thrown violently against this bone it is liable to be torn and contused. The temporo-sphenoidal lobe lies upon the very rough and irregular petrous bone, but the posterior lobes of the brain lie upon the tentorium; consequently, the posterior lobes are much the less likely to be injured. In proportion to the injuries to the brain so the patient exhibits evidence of the disturbance it has caused. He suffers from " concussion," but that is a vague term, which should be used by you merely as a clinical expression to indicate a series of clinical symptoms; it is not at all an accurate pathological term and it does not imply any one definite lesion. Where the patient suffers from "concussion" there is, as a rule, contusion or laceration of the brain, and often its superjacent membranes are damaged. What are the symptoms of concussion? In general terms one may say they commence with a certain amount of shock. You know that when an important part of the body is injured shock results. That is eminently true of the abdominal and thoracic viscera, as well as of the viscus inside the skull. The temperature is liable to be low, the patient becomes cold, and in the case of the woman whom I have several times referred to, her temperature was $95.4^{\circ}$. The man now in the hospital who has probably an injury to his brain had a temperature just over $96^{\circ}$. Very often the skin of a patient suffering from shock is clammy and the vital powers all seem to be at a low level; the pulse is feeble, the blood stream may be slow or irregular and the muscular system is also more or less feeble - the man cannot stand or sit up. Cerebration is also slow. If the patient is not completely unconscious and you ask him quite a simple question, such as his name, the answer will be given only after a definite interval. He may be able to tell you where he lives, or he may not. Every muscular effort is feeble and made with difficulty. Between such a condition and absolute unconsciousness there is every possible gradation. When a man is completely unconscious and cannot be aroused at all you argue that he has had a very severe injury to his brain. The length of time during which a patient may remain unconscious varies enormously. In a case of very slight injury to the brain the unconsciousness may be very transient, in other cases it may be days, weeks, or even months before he regains his ability to explain what he wishes or to express his feelings in proper words.

Let us refer for a moment to another man who was in Abernethy Ward, and who illustrates some of the points I have been speaking of. He illustrates also some of the later complications. This patient was admitted two years ago, after an injury to his skull. We diagnosed that he had a fracture of the anterior fossa, for these reasons: $\mathrm{He}$ had an enormous flow of blood externally through his nose, through his mouth, and into his stomach, so that, apart from what he lost externally, he vomited up about 2 pints of blood within a very short time of his accident. After that we found that he had great extravasation all round and in both orbits. There was no evidence of escape of cerebro-spinal fluid nor of injury of nerves. He was absolutely unconscious, and could not be roused in any way at all. He was suffering from extreme shock, his pulse could hardly be felt, he was cold, and appeared to be very nearly dead. It was days before we could feel that he was likely to survive, and for three weeks he was quite unconscious. That does not mean to say that he did not move his limbs, but he was unconscious of his surroundings. It was only by very careful nursing and feeding that he was kept alive. After three weeks he slowly recovered consciousness, and, like all those people who have had concussion and injury to the brain, he had at first headache and giddiness, and these symptoms were aggravated by the slightest effort. $\mathrm{He}$ had also no recollection of his accident.

It is a characteristic of all cases of severe injury to the brain in which there is more than momentary insensibility, that a man forgets what happened directly before the accident. Thus, a patient was brought into hospital who had fallen from a staging on a building, and.who had the symptoms of concussion of the brain and was quite unconscious. He soon came round after being put to bed, and was able to tell us that he had fallen from a building on which he was working. When we came to question him, we found that the last thing he remembered was going up a ladder, and he believed he had fallen from this ladder. Inquiry showed that this was not so, that he had climbed up to the top of this ladder, that he had got on to the staging, and that afterwards when he was on this staging which had been insecurely fastened, it tilted and precipitated him to the pavement below. So the last portion of time immediately before his accident was quite obliterated from his memory. Take another case which illustrates very well a longer period of loss of memory. A man came into the hospital having been brought from Holborn Viaduct, where he was pitched off a van, the horse having bolted. The wheel of the van came into contact with one of the lamp-posts and he was precipitated into the road on to his head. He remained more or less unconscious for two days. After he had come to himself we began to question him about the occurrence. He told us that the accident must have happened in the Borough, for that was the last place he remembered passing through. But it turned out, from independent evidence, that after being in the Borough he had driven over London Bridge and through the City, and it was while driving westwards on that journey that he met with the accident. All the recollection of what happened after he left the Borough was obliterated, and he lost in time about three-quarters of an hour. In another case a man was brought into hospital with severe injury to his head due to the breaking of a grindstone while it was revolving at a rapid rate. He had a lacerated wound, with fracture of his skull and injury to his brain. When he came to himself we found he was under the impression that the day of the week on which the accident occurred was Monday, when, as a matter of fact, it was Saturday. The only thing which he remembered was going to work on Monday, the remaining days having been completely forgotten. The memory of what has happened immediately before the accident is, as a rule, never restored, and that is an important matter, because a man may quite honestly give you an utterly erroneous history. There is also, in these people, a strange perversion of the perception of what has happened, so that facts, and important facts, may not only have been forgotten, but those which the victim of the accident remembers may be quite perverted or distorted.

Well, the man who had this three weeks' insensibility slowly recovered, and after many weeks he was well enough to leave the hospital. A year afterwards he came back to tell us that he had had an epileptic fit. During that year he had apparently been tolerably well. He had returned to his work, though since the accident his work had been of a lighter character than that to which he had previously been accustomed. Still he had been earning his own livelihood. And then, without any warning, an acute, violent epileptic fit came on. This was a very serious occurrence, and the prognosis was serious also. I put him upon considerable doses of bromide of potassium, and kept him under its influence for weeks and months. But after a few months he had another fit, and after a shorter interval still another, and so on, so that when I last saw him, in the summer, those fits had become increasingly frequent. The prognosis in his case has become extremely grave, because it is quite likely that in the end the man will become insane. And this man's case shows that in injury to the head, when you have seen the patient out of the hospital you have not necessarily seen the last of him, for after a man has had an injury to his brain he is liable to suffer for long from an irregular and easily-upset cerebral circulation. There are two things 
which you ought always to warn these people about, and which I do constantly warn them about, and the first is that the least quantity of alcohol will make them completely intoxicated. You know that the effect of alcohol is to cause flushing of the surface of the brain, and this result is brought about by even the smallest quantities of alcohol in many of these people who have had brain injury. So if there is one condition more than another which requires that a man shall be a teetotaler, it is serious injury to the brain, with perhaps prolonged insensibility, from which he has been fortunate enough to recover. The other thing against which I warn patients is violent over-exertion, and especially exposure to a hot sun. A man who has once had sunstroke is very liable to have headache and a return of his symptoms if he again exposes himself to the sun or over-exerts himself.

And the man with an injured brain is in a similar condition. The state of his cerebral circulation makes him very liable to have headache of a severe type, with giddiness, nausea, and impaired mental ability and control. And remember that these sequelae often last many years.

Now, you will naturally ask me, What is there inside the head of this man with traumatic epilepsy which has brought about this serious condition? If you could look inside the head, what would you see? Let me remind you of what I told you we saw in the case of the man who died a fortnight after his fracture of the base of his skull. I told you he had haemorrhage between the dura mate and the bone, and that he had extravasation of blood underneath the pia mater and tearing of the temporosphenoidal lobe. Well, either of these conditions may result in some permanent irritation of the cortex of the brain, and you may be sure that the man who has developed epilepsy, and who was so long unconscious, had an extensive tear of his brain and that he had extravasation of blood in large quantities. He probably had extensive scarring of the torn tissues and a hardening and thickening of the membrane called pachymeningitis which has caused irritation of the surface convolutions resulting in the epileptiform fits. Unfortunately for the patient there is not the least evidence to help us as to where he has this trouble, for there is no localizing evidence. ' In some cases the evidence is clear. Sometimes the epilepsy is of the "Jacksonian" type, in which the fit begins always by a convulsion or spasm in some particular portion of the body, and spreads from thence until it becomes generalized. So that a man with Jacksonian epilepsy may have a fit commencing by spasmodic movements in the thumb which may spread to his fingers and then to the forearm, then to the arm, and so to his body. In that case he has what is called a focal lesion, for there is a point in the brain from which this trouble spreads, and the part affected in such a case as I have suggested is the cortical centre for the thumb and the hand. In that locality there may be a piece of displaced or jagged bone, or there may be a blood cyst, or some thickening of the membranes or some scarring of the brain. If there are any of these indications you may accept them as suggestions for a mode of treatment which may save the patient. You may operate at the place which is indicated by the position and character of these spasms, and you may remove a portion of the bone which may be pressing on the brain, or you may evacuate blood which has accumulated and is exerting similar pressure. I will conclude by mentioning one more case to illustrate this condition. A man had an injury to his skull and brain for which he was admitted into another hospital, and there he remained semi-conscious or unconscious for nearly three months. He recovered from this injury very slowly, and afterwards, as people say, he was never the same man as before. Instead of being cheerful and good-tempered, he was morose and unsociable, and became liable to violent fits of passion, which were purposeless and apparently causeless. These attacks continued, and were succeeded at the end of more than a year by epilepsy, and the epileptic attacks rapidly increased in frequency till he was brought to this hospital in a condition of what is called post-epileptic coma. In this condition he died. We examined him post mortem, and found that on one side of his brain there was a large blood cyst. There had been extravasation into the subarachnoid space of blood from either a torn brain or torn membranes, and the blood had become encysted, and caused irritation to the cortex of his brain. I think if he could have been operated upon that man's life might have been completely altered, for it is likely that the whole of his symptoms were due to the pressure and irritation of this blood cyst.

\section{INTRAPERITONEAL IMPLANTATION OF THE URETERS INTO THE COLON.*}

By GILBERT BARLING, M.B., F.R.C.S., SURGEON TO THE GENERAL HOSPITAL : PROFESSOR OF SURGERY IN THE UNIVERSITY OF BIRMINGHAM.

Few conditions are more distressing than the constant escape of the whole of the urine above the pubes with practically no efficient means of control. Most of the patients who suffer from this misery are the subjects of extroversion of the bladder, and though life to some of them may be made more or less tolerable, yet the results of the various plastic operations are, on the whole, so unsatisfactory, that I am sure most surgeons dread having such cases committed to their charge.

In recent times, for the relief of extroversion, there has been a tendency to divert the urine into the large bowel as an alternative to direct plastic operations, with their disappointing results. This is the revival of a proeedure first imperfectly carried out by Sir John Simon, and later, on a sounder plan, by Sir Thomas Smith. The latter surgeon formulated the extraperitoneal method of implantation of the ureters each into the colon of its own side through a lumbar incision; this in 1878. The final result of this operation, however, was discouraging, and, as far as I am aware, several years elapsed before the method was revived, and with it others on similar lines. At the present time, in addition to the extraperitoneal method of operation, we have two others-(1) intraperitoneal implantation of each ureter separately into the colon of its own side; and (2) implantation of the separated trigonal area with both ureteral orifices into either rectum or sigmoid.

Diversion of urine into the bowel may be resorted to for other conditions than extroversion of the bladder. In cancer of the bladder encroaching, as it is apt to do, on the trigonal region, ex cision of the growth may be feasible if provision can be made for the collection and storage of the urine after removal of the lower ends of the ureters in the resection.

In accidental division of ureters in abdominal operations, when re-implantation into the bladder is impossible, grafting into the colon is worth consideration as an alternative to nephrectomy.

Another extension may be found in unfortunate patients such as the one I exhibit to you to-day-the victim of an injury :

This young man, aged 20 years, was, five years ago, admitted to a hospital suffering from fractured pelvis with ruptured urethra and laceration of the neck of his bladder. Exit for the urine was given by suprapubic drainage, and when I saw the patient on June 4th, 1906, the whole of the urine escaped above the pubes, where there was, in addition, urine escaped above the pubes, where there was, in addition, a suppurating sinus leading down to the back of the fractured pubic bone. A appears to have resulted from the injury. for though the age of puberty has been attained, the penis and testicles are quite undeveloped, as are the other sexual characters.

As no effort had hitherto been made to restore the urethra, I thought this should be attempted, but the final result was in some degree an exaggeration of the patient's miseries, for a recto-vesical fistula was formed, and the treatment was abandoned. At the time of this operation the suprapubic wound was opened up, and the densest 'oi scar tissue was found under the pubic arch and encroaching upon the neck of the bladder.

The failure to secure patency of the urethra led me to adopt ureteral implantation for the patient's relief. The three methods which I have already mentioned were open to me. Maydl's operation, implantation of the trigonal area with both ureters into the rectum, which has much to recommend it in cases of extroversion, was here quite unsuitable owing to the dense matting of parts already referred to. For each of the remaining alternatives there were arguments. The extraperitoneal operation avoids

* A note read at the meeting of the Clinical and Pathological Section of the Birmingham Branch on October 26th, 1906. 